\title{
Implantação do Processo Aquisição no Contexto do Processo de Desenvolvimento de Software na Synapsis Brasil
}

\author{
Carlos Simões ${ }^{1,2}$, Gleison Santos ${ }^{3}$, Ana Regina Rocha ${ }^{2}$ \\ ${ }^{1}$ Synapsis Brasil \\ Av. das Américas no 3434, Bloco 2, Sala 403 \\ Barra da Tijuca - CEP 22640-102 - Rio de Janeiro - RJ \\ ${ }^{2}$ COPPE/UFRJ - Universidade Federal do Rio de Janeiro \\ Programa de Engenharia de Sistemas e Computação \\ Av. Horácio Macedo, 2030, Prédio do Centro de Tecnologia, Bloco H, Sala 319 \\ Caixa Postal 68511 - CEP 21941-914 - Rio de Janeiro, RJ \\ ${ }^{3}$ UNIRIO - Universidade Federal do Estado do Rio de Janeiro \\ Departamento de Informática Aplicada - CCET \\ Avenida Pasteur, 458 - Urca - Rio de Janeiro/RJ - CEP 22290-240 \\ cs@synapsisbrasil.com.br, \{carlossimoes, darocha\} dcos.ufrj.br, \\ gleison. santosauniriotec.br
}

\begin{abstract}
The execution of software projects sometimes is only viable if some activities are outsourced. Thus, the definition and execution of software acquisition process are important to enhance project's control and quality. This paper presents the Acquisition process implementation at Synapsis Brasil in accordance to MR-MPS and CMMI. We also discuss the integration between the acquisition process and the software development process. Finally, the main benefits, difficulties, success factors and lessons learned are discussed.

Resumo. A execução de um projeto de software às vezes se mostra mais viável com a terceirização de algumas das etapas. Devido a isto, a definição e execução de um processo de aquisição de software são importantes visando ao aumento do controle e da qualidade do projeto. Este artigo apresenta a adoção do processo de aquisição durante a iniciativa de melhoria de processo mais recente da Synapsis Brasil, em conformidade com o MR-MPS e CMMI. É destacada a integração do processo de aquisição com o de desenvolvimento de software. Também são identificados os principais benefícios e dificuldades encontradas, fatores de sucesso e lições aprendidas.
\end{abstract}

\section{Introdução}

Com a exigência da qualidade aumentando por parte dos clientes, as organizações desenvolvedoras de software reconhecem que tratar a questão da qualidade de forma mais profissional, investindo em treinamento, processos, técnicas e ferramentas que permitam a melhoria da qualidade é de fundamental importância. Atrelada à questão da exigência pela qualidade está a necessidade cada vez maior do cliente em receber o produto em um prazo cada vez menor. Nem sempre é possível, no entanto, ter todas as condições necessárias para o desenvolvimento total do produto na própria empresa. Muitas vezes é necessária a terceirização de parte das atividades. Porém, a terceirização não é simples, existindo grande quantidade de casos de insucessos por diversos motivos, sejam por as- 
pectos técnicos, sociais, administrativos, comunicação entre outras.

A melhoria de processo de software assume que uma organização bem gerenciada com indicadores e processos definidos tem mais possibilidades de produzir produtos que atendem mais adequadamente aos requisitos dos clientes, no prazo e no orçamento do que organizações mal gerenciadas e sem processos (Solingen e Berghout, 1999). Controlar qualidade, produtividade e prazo tanto de contratada quanto de contratante sem a adoção de modelo de qualidade de processo de software é uma tarefa muito difícil. A Synapsis Brasil tem investido em melhoria de processos ao longo do tempo e, recentemente, implantou o nível C do MR-MPS (Softex, 2009) e nível 3 do CMMI (Chrissis et al., 2006), sendo avaliada com sucesso em junho e julho de 2009, respectivamente. Dentro do escopo dessa iniciativa de melhoria, foi definido e implantando um processo de Aquisição fazendo com que a Synapsis tenha sido a primeira empresa no Brasil a ser avaliada no Processo Aquisição do MR-MPS.

Este artigo apresenta a adoção do processo de aquisição na Synapsis Brasil, em conformidade com o MR-MPS e CMMI, e está divido em 3 seções além da introdução: na seção 2 são comentadas as etapas da iniciativa de melhoria na Synapsis Brasil; a seção 3 contém a estratégia de implantação utilizada e a seção 4, as considerações finais.

\section{Melhoria da Qualidade na Synapsis Brasil}

A Synapsis Brasil é uma organização de médio porte cuja missão é satisfazer as necessidades de serviços relacionados à Tecnologia da Informação, Telecomunicações e Telecontrole para as empresas que constituem o grupo Endesa e também para clientes externos. A Synapsis Brasil adotou como um de seus objetivos estratégicos o investimento contínuo na melhoria da qualidade de seus produtos e serviços. Assim, foi elaborado o Plano Estratégico da Qualidade alinhado com as estratégias corporativas da Matriz localizada em Santiago, Chile, englobando os seguintes objetivos: ISO 9001:2000 (março de 2005); CMMI Nível 2 (agosto de 2006); MPS.BR nível C (junho de 2009); CMMI nível 3 (julho de 2009); e MPS.BR nível A e CMMI nível 5 (Junho de 2012).

O plano tem como particularidade (i) englobar duas unidades (Niterói e Fortaleza), com processos comuns e implantados ao mesmo tempo, e (ii) a grande separação geográfica. Isto torna necessária uma integração muito grande das duas equipes e um gerenciamento centralizado e eficaz de modo a fazer com que as unidades caminhem em sintonia. O conhecimento precisa ser disseminado de forma igual nas duas unidades. Além disso, a motivação e comprometimento das equipes devem ser os mesmos.

Para apoiar a implementação do processo Aquisição e também dos outros processos necessários ao nível C de maturidade do MPS.BR (Softex, 2009), foram definidos os seguintes grupos organizacionais: (i) Grupo de Processo, com o objetivo de definir os processos; (ii) Grupo de Garantia da Qualidade do Processo e do Produto, com objetivo de avaliar a qualidade dos produtos gerados e também a aderência dos processos sendo executados em relação aos processos padrão da empresa; (iii) Grupo de Gerência de Configuração, responsável por avaliar, executar e assegurar a correta implementação da Gerência de Configuração; (iv) Grupo de Métricas, responsável por toda medição e análise de dados da empresa, o que inclui análise das métricas, comunicação das métricas à alta direção, entre outros. Foram realizados treinamentos e workshops relacionados às áreas de processos que seriam implementadas para os colaboradores da empresa. Depois 
disso, os projetos usando o processo tiveram início.

Para apoiar a definição, implantação e execução das atividades, os consultores da COPPE/UFRJ foram considerados fator crítico de sucesso. A realização das atividades do processo era acompanhada por um consultor experiente. Essa abordagem foi eficiente para treinar as equipes da organização nos processos, ferramentas e nas melhores práticas da Engenharia de Software. Também foi importante para fazer com que os profissionais se conscientizassem dos benefícios de se usar um processo de software definido. A gerência de alto nível também teve papel fundamental no projeto de melhoria ao deixar claro para todos que a iniciativa de melhoria era de grande importância e prioridade para a organização. Todos se envolveram de alguma forma visando ao sucesso do projeto: gerentes de projetos, líderes de projetos, analistas, testadores, equipe de qualidade, programadores, e outros.

\section{Estratégia de Implantação do Processo Aquisição}

O propósito do processo Aquisição é gerenciar a aquisição de produtos e/ou serviços que satisfaçam a necessidade expressa pelo adquirente, tendo como objetivo assegurar a qualidade do produto de software, independentemente de parte ou todo estar sendo desenvolvido externamente à equipe do projeto. O processo Aquisição no MR-MPS (Softex, 2009) é composto de nove resultados esperados, focando na seleção do fornecedor e no acompanhamento dos produtos (avaliação periódica dos produtos intermediários e finais) e processos (como o desenvolvedor está executando o desenvolvimento do produto). Para apoiar controles e avaliações necessários, todos os aspectos para assegurar a qualidade dos produtos resultantes do processo Aquisição devem estar claramente definidos no contrato entre as partes.

Devido à particularidade dos projetos da Synapsis em que nem todos os projetos contemplam a contratação de fornecedores, optou-se por não incluir as atividades do processo de aquisição dentro do processo de desenvolvimento de software. Com isto, quando um projeto não contemplar aquisição, o líder do projeto não necessita solicitar a não execução destas atividades. Ao ser definida a necessidade de aquisição em um projeto, o processo Aquisição deve ser executado em paralelo com o processo de desenvolvimento. A decisão da não integração levou em consideração os seguintes fatores: (i) Nem todos os projetos possuem aquisição; (ii) A aquisição pode ser referente a determinados produtos do processo de desenvolvimento de software. O escopo da aquisição contido no plano de aquisição estabelecerá o que será adquirido, podendo ser: Especificação dos requisitos do produto; Elaboração da especificação de técnica; Codificação e testes de Unidade; e (iii) Mesmo quando o projeto tem aquisição, não necessariamente todas as funcionalidades do produto precisam ser adquiridas. Como exemplo, pode-se optar pela aquisição somente de funcionalidades implementadas em uma linguagem de programação para a qual não haveria recursos humanos disponíveis ou com conhecimento necessário.

A necessidade de aquisição pode ser identificada em qualquer ponto do processo de desenvolvimento. Por exemplo, caso seja identificada a necessidade de adquirir a "Especificação dos requisitos do produto", o processo de aquisição deve começar a ser executado na fase de Planejamento do processo de desenvolvimento de software. Durante os monitoramentos do projeto, ao longo das fases seguintes, o líder do projeto avalia a 
necessidade de aquisição. Caso seja identificada a necessidade de aquisição (Especificação Técnica e/ou Construção), é iniciada a execução do processo de aquisição. A contratação de aquisições pela Synapsis é realizada na forma de preço e escopo fechado quando se trata da terceirização de todo ou parte do desenvolvimento. Isto é, elabora-se a especificação dos requisitos do produto (com lista de requisitos especificada e projeto de arquitetura definido), faz-se a estimativa de pontos de função para definir o tamanho do produto e solicita-se cotação ao fornecedor. O pagamento é feito por entrega do produto contratado. Negociações sobre alteração de escopo (mudança de requisitos) ou crescimento do tamanho do produto são realizadas em comum acordo.

\subsection{O Processo de Aquisição}

Um esquema simplificado do processo Aquisição pode ser visto na Figura 1 (Simões et $a l ., 2009)$ e contempla as atividades relacionadas diretamente com o planejamento e execução do plano de aquisição, enquanto o processo de desenvolvimento de software contempla as atividades de apoio e engenharia de software. Portanto, alguns tópicos ou artefatos não são descritos no processo de aquisição, existindo apenas referências aos artefatos que são elaborados no processo de desenvolvimento.

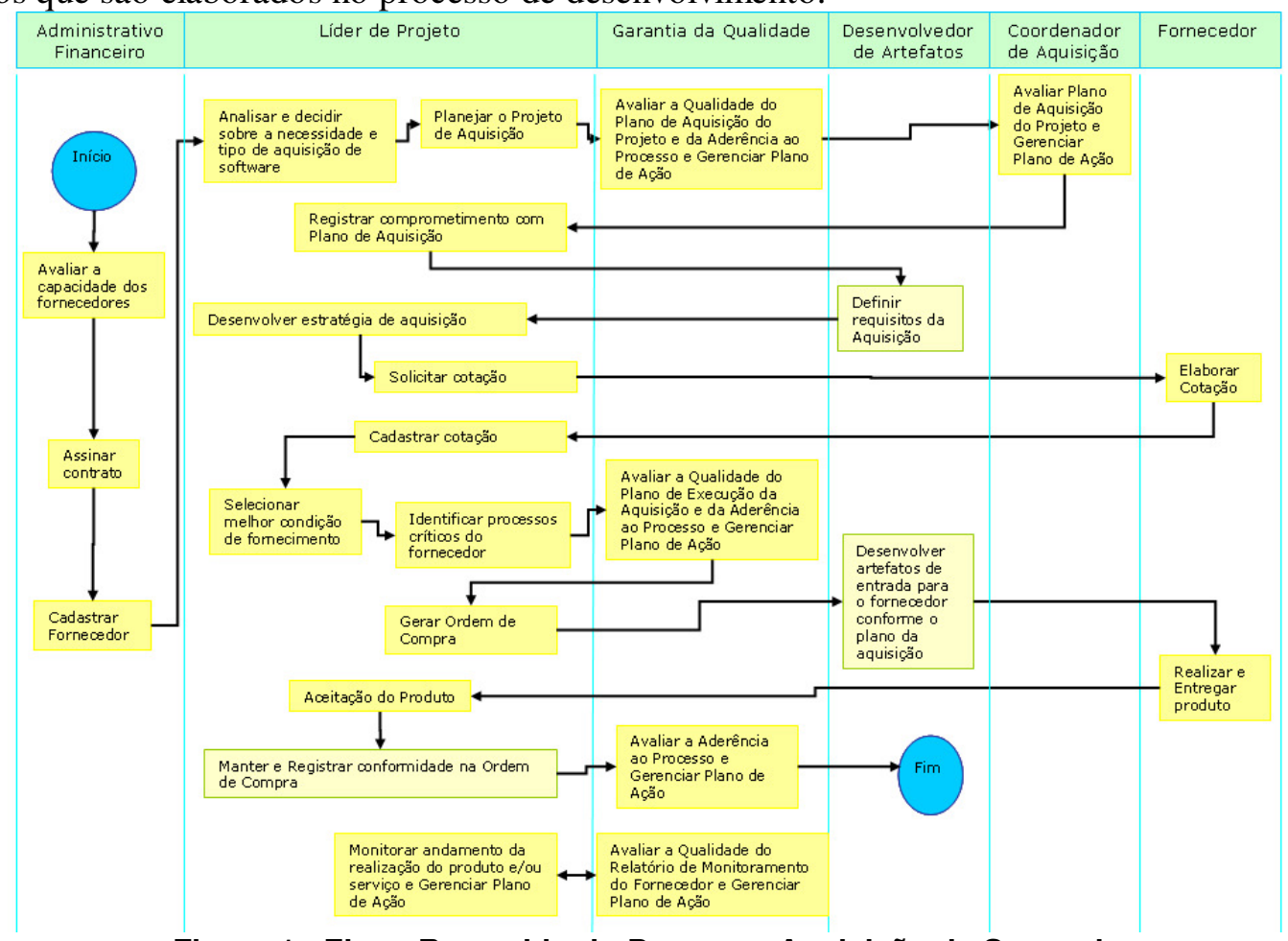

Figura 1 - Fluxo Resumido do Processo Aquisição da Synapsis

Com o objetivo de guiar o líder do projeto na execução do processo Aquisição, foram incluídos pontos de controle, como, por exemplo, na apresentação da pré-venda do projeto para a diretória e na elaboração do relatório de monitoramento do projeto. $\mathrm{O}$ processo Aquisição definido deve ser adaptado a cada execução, dependendo do contexto da execução e dos objetivos da aquisição. Este processo pode ser executado em dois contextos: organizacional e em projetos de desenvolvimento de software.

No contexto organizacional, é mantido o cadastro de fornecedores, que pode ser 
elaborado em duas situações: (i) Para avaliar um conjunto de potenciais fornecedores, firmar contratos e incluir os selecionados no cadastro de fornecedores da Synapsis; (ii) Para avaliar um único fornecedor, firmar contrato e incluí-lo no cadastro de fornecedores da Synapsis. Em quaisquer destas situações, o processo de aquisição deve ser adaptado contendo obrigatoriamente as macro-atividades: (i) "Manter Cadastro de Potenciais Fornecedores" (cujas tarefas associadas são "Avaliar a capacidade dos fornecedores", "Assinar contrato" e "Cadastrar Fornecedor"); (ii) "Avaliar a Qualidade dos Produtos do Processo" (não representada na Figura 1 por restrições de espaço); (iii) "Avaliar Aderência ao Processo" (idem anterior).

No contexto de projetos de desenvolvimento de software, a execução é iniciada quando se avalia e toma-se a decisão de contratar fornecedor para desenvolvimento do produto ou adquirir COTS (Commercial-of-the-Shelf - pacotes). Neste caso, o processo de aquisição deve ser adaptado contendo obrigatoriamente as seguintes macroatividades: (i) "Avaliar a Qualidade dos Produtos do Processo" (diversas variantes podem ser vistas na Figura 1); (ii) Preparar Aquisição de Software (inclui as atividades do fluxo desde "Analisar e decidir sobre a necessidade e tipo de aquisição de software" até "Registrar comprometimento com Plano de Aquisição"); (iii) "Concluir Preparação para a Aquisição de Software" (inclui de "Definir requisitos da Aquisição" a "Cadastrar cotação"; (iv) "Selecionar Fornecedores" (inclui de "Selecionar melhor condição de fornecimento" a "Gerar Ordem de Compra". No caso de aquisição de COTS, caso pertinente, a atividade "Identificar processos críticos do fornecedor" pode ser excluída); (v) "Monitorar andamento da realização do produto e/ou serviço e Gerenciar Plano de Ação" (no caso de aquisição de COTS, caso pertinente, esta macro-atividade pode ser excluída); (vi) "Aceitação do Produto" (prevê, também, a avaliação da qualidade do produto entregue pelo fornecedor e o respectivo aceite por parte da Synapsis Brasil); (vii) "Avaliar Aderência ao Processo" (diversas variantes podem ser vistas na Figura 1).

\subsection{Execução do Processo de Aquisição}

Para todo projeto que necessita adquirir partes de seus componentes, deve ser elaborado o plano de aquisição do projeto considerando o escopo das necessidades a serem contempladas. Além disso, deve-se planejar o processo, identificar responsabilidades, cronograma e riscos, planejar gerência de documentos e de comunicação. Tal plano inicia com a atividade "Analisar a Necessidade de Aquisição de Software" em que são analisadas as necessidades, confirmado ou não ser objeto de aquisição e os resultados que se pretende atingir com a aquisição. Se for constatado, por algum motivo, a não realização da aquisição, este processo é encerrado.

Caso seja confirmada a necessidade ou interesse em realizar a aquisição, a atividade "Analisar a Necessidade de Aquisição de Software" é realizada com objetivo de analisar as necessidades a serem atendidas, avaliar e decidir o que deve ser adquirido, os resultados a serem atingidos e a indicação do que será contratado, isto é, o escopo e o tipo de aquisição. Pode-se tomar uma das seguintes decisões: (i) Contratar desde a especificação de requisitos; (ii) Contratar a partir da especificação técnica; (iii) Contratar a construção; (iv) Adquirir COTS. Ao final, é produzido um documento com o conjunto de necessidades a serem contempladas pela aquisição, o escopo e o tipo de aquisição.

As funcionalidades contidas no documento "Plano de Aquisição do Projeto" de- 
vem estar descritas em detalhes no artefato "Especificação dos requisitos do produto" aprovado previamente pelo cliente. Com o plano de aquisição aprovado, o passo seguinte é verificar potenciais fornecedores e decidir para quais serão solicitadas cotações. Ao receber as cotações dos potenciais fornecedores, inicia-se a seleção do fornecedor a partir dos potenciais fornecedores e da resposta à solicitação de cotação. Durante a execução desta atividade, poderão ser feitas negociações com os fornecedores.

Para a elaboração do artefato de apoio a seleção da melhor oferta, foi utilizado o processo de Gerência de Decisão (GDE) (Softex, 2009). Busca-se, assim, reduzir a subjetividade da decisão e, desta forma, espera-se uma maior probabilidade de selecionar uma solução que atenda às múltiplas demandas dos envolvidos. Exemplos de critérios utilizados são: experiência anterior em trabalhos similares; desempenho anterior em trabalhos similares; capacidade de gerenciamento; disponibilidade de recurso; disponibilidade de infra-estrutura; habilidade em trabalhar como fornecedor; risco associado; facilidade de comunicação; maturidade do processo do fornecedor; densidade de defeito em trabalhos anteriores; desvio de prazo em trabalhos anteriores; desvio de esforço em trabalhos anteriores; facilidade de negociação; facilidade de correção de defeitos; nível de conhecimento do processo da Synapsis, além de prazo de entrega e valor da oferta.

Finalizando a decisão por um fornecedor, é elaborada a ordem de compra ou aditivo, em caso de contrato guarda-chuva, e iniciado o monitoramento das atividades com o fornecedor como estabelecido no contrato. Estas atividades são realizadas periodicamente pelo líder de projeto durante a execução da atividade de monitoramento do projeto. Ao ser entregue o produto adquirido, são solucionadas quaisquer questões relacionadas à aceitação, de acordo com os procedimentos estabelecidos na ordem de compra durante a execução das atividades "Integrar o Produto e Testar a Integração" e "Testar o Produto" do processo de desenvolvimento de software. Depois de sanados problemas, a conformidade do produto / serviço na Ordem de Compra é registrada. Ao final do projeto é feita uma avaliação dos serviços prestados pelo fornecedor para realimentar as informações do cadastro de fornecedores da Synapsis.

\subsection{Interação do Processo de Aquisição com o de Desenvolvimento de Software}

No processo de desenvolvimento definido pela Synapsis algumas atividades têm a identificação do momento no qual cada atividade do processo de aquisição deve ser executada. As atividades existentes no fluxo do processo de Aquisição (ver Figura 1) marcadas com (*) possuem correspondência com atividades do processo de desenvolvimento, a marcada com $(* *)$ tem correspondência com várias atividades do processo de desenvolvimento, sendo executada por diferentes perfis conforme a estratégia de aquisição estabelecida. As atividades de desenvolvimento que têm interação com o processo de aquisição, isto é, nas quais pode ser constatada a necessidade de aquisição ou onde a existência de aquisição deve ser levada em consideração, são apresentadas na Tabela 1.

Tabela 1 - Relacionamento entre Atividades de Desenvolvimento e de Aquisição

\begin{tabular}{|l|l|}
\hline $\begin{array}{l}\text { Elaborar escopo e } \\
\text { estimativas do } \\
\text { projeto }\end{array}$ & $\begin{array}{l}\text { Primeiro momento onde é possível identificar a necessidade de aquisição para o } \\
\text { projeto e interação com o grupo de reutilização para indicar o uso de um ativo } \\
\text { reutilizável ou submeter novos ativos a candidatos à reutilização. }\end{array}$ \\
\hline $\begin{array}{l}\text { Elaborar e conso- } \\
\text { lidar plano do } \\
\text { projeto }\end{array}$ & $\begin{array}{l}\text { Poderá ser elaborado o Plano da aquisição (se pertinente), é descrito o escopo, o } \\
\text { conjunto de necessidades a serem contempladas pela aquisição e o tipo de aquisi- } \\
\text { ção a ser contratado. Neste plano também são identificados responsabilidades, }\end{array}$ \\
\hline
\end{tabular}




\begin{tabular}{|c|c|}
\hline & ronograma, riscos e gerência de documentos e de comunicação. \\
\hline $\begin{array}{l}\text { Monitorar anda- } \\
\text { mento do projeto } \\
\text { - Fase } 2 \text { (Especi- } \\
\text { ficação de Requi- } \\
\text { sitos) e Fase } 3 \\
\text { (Desenho, Cons- } \\
\text { trução e Testes) }\end{array}$ & $\begin{array}{l}\text { Durante os monitoramentos realizados na Fase } 2 \text {, o líder de projeto deverá reava- } \\
\text { liar a necessidade de contratação de fornecedor para a execução de partes do } \\
\text { projeto. Caso seja identificada a necessidade, o processo de aquisição tem sua } \\
\text { execução iniciada (planejamento e execução da aquisição). Se foi contratado um } \\
\text { fornecedor para parte do produto, isto é, já foi iniciada a execução do processo de } \\
\text { aquisição, o líder de projeto deverá, também, monitorar o processo de aquisição } \\
\text { com um intervalo de uma semana. Na elaboração dos relatórios de monitoração } \\
\text { são registradas informações habituais do projeto como desvios e alterações de } \\
\text { planos e, também, informações específicas dos fornecedores como a qualidade dos } \\
\text { produtos intermediários entregues, o cumprimento dos prazos acordados e outros } \\
\text { itens identificados nos acordos estabelecidos (por exemplo, a forma de } \\
\text { monitoração dos processos do fornecedor críticos para o produto final). Durante os } \\
\text { monitoramentos realizados na Fase } 3 \text {, o líder de projeto deverá reavaliar a } \\
\text { necessidade de contratação de fornecedor para a execução de partes do projeto. } \\
\text { Caso haja necessidade, o processo de aquisição tem sua execução iniciada (se não } \\
\text { tiver sido iniciado na fase anterior). }\end{array}$ \\
\hline $\begin{array}{l}\text { Avaliar a quali- } \\
\text { dade do relatório } \\
\text { de monitoração } \\
\text { do projeto pelo } \\
\text { PPQA - Fase } 2 \text { e } \\
\text { Fase } 3\end{array}$ & $\begin{array}{l}\text { Nesta atividade é realizada a avaliação da qualidade do relatório de monitoração } \\
\text { do projeto de acordo com os critérios estabelecidos no laudo de avaliação pelo } \\
\text { PPQA (membro do Grupo de Qualidade). Caso tenha sido identificada a necessi- } \\
\text { dade de aquisição, o PPQA deve avaliar os produtos e o processo relativos a aqui- } \\
\text { sição. Para as não conformidades deve ser gerado um plano de ação que deve ser } \\
\text { gerenciado de forma a garantir a sua execução. }\end{array}$ \\
\hline $\begin{array}{l}\text { Avaliar a aderên- } \\
\text { cia aos processos } \\
\text { - Fase } 2 \text { e Fase } 3\end{array}$ & $\begin{array}{l}\text { Durante a execução desta atividade, o PPQA, se pertinente, deverá também avaliar } \\
\text { a aderência ao processo de aquisição. }\end{array}$ \\
\hline $\begin{array}{l}\text { Analisar desem- } \\
\text { penho do projeto } \\
\text { e da equipe }\end{array}$ & $\begin{array}{l}\text { Se ocorrer aquisição, o líder de projeto deve avaliar a capacidade dos fornecedo- } \\
\text { res, isto é feito com o apoio do artefato "Avaliação de desempenho do fornecedor". } \\
\text { Após a elaboração deste artefato, o líder de projeto deve enviá-lo para a alta ge- } \\
\text { rência que decidirá se o fornecedor será mantido no cadastro ou excluído. }\end{array}$ \\
\hline
\end{tabular}

\subsection{Resultados Obtidos com a Execução do Processo de Aquisição}

O processo de Aquisição foi executado pela primeira vez em um projeto levado às avaliações CMMI e MPS. Os resultados foram satisfatórios, tornando-se, assim, padrão para todas as aquisições de software na Synapsis Brasil. O monitoramento de indicadores de desempenho atrelados ao Plano de Medição facilitou o entendimento da execução do processo. A Figura 2 apresenta indicadores referentes à execução em um projeto com 63 pontos de função e implementado em Java. Este tamanho foi considerado adequado pois facilita o gerenciamento e possibilita respostas em um curto período de tempo.

Os indicadores coletados a analisados foram o "Índice de Desempenho de Prazo" (IDP) e o "Desvio de Prazo do Fornecedor" (DPET). O ideal seria que ambos apresentassem valor 1 ou muito próximo disso. $\mathrm{O}$ resultado foi considerado animador, com indicadores dentro dos limites estabelecidos pela Synapsis. O pequeno desvio de prazo identificado foi em função do entendimento e adequação do fornecedor ao novo modelo de processo de Aquisição e aos templates utilizados. Observa-se que após o período inicial não ocorreu mais desvio de prazo do fornecedor. Demonstrando que, além do processo de Aquisição, os monitoramentos e a utilização dos artefatos foram fatores de sucesso.

Ainda não foram executados projetos que necessitassem de COTS. Dessa forma, uma parte do processo de Aquisição não pode ser avaliada. Espera-se após executar o processo envolvendo aquisição de COTS ele possa ser refinado e os templates adequa- 
dos (por exemplo, definindo critérios para identificação e seleção de produtos).
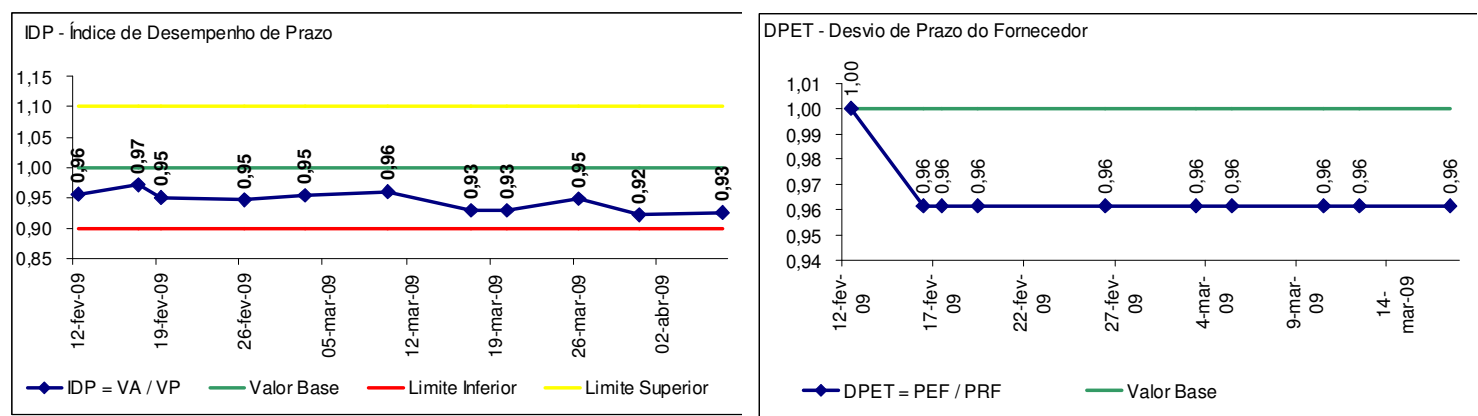

Figura 2 - Índice de Desempenho de Prazo e Desvio de Prazo do Fornecedor

\section{Considerações Finais}

A avaliação dos processos na Synapsis foi conduzida pela Riosoft (MPS.BR) e Crest Consulting (CMMI). A avaliação inicial foi feita em conjunto e as avaliações finais em separado com 3 semanas de intervalo. Nenhuma fraqueza substancial foi identificada. Durante a implantação dos processos, todos os envolvidos, depois de pouco tempo, buscavam aprender e melhorar suas próprias atividades, uma vez que houve uma percepção geral de que o uso de um processo definido melhorou a forma de trabalho na empresa.

Atrelada à exigência pela qualidade está a necessidade cada vez maior do cliente em receber o produto em um prazo cada vez menor. Como a demanda por desenvolvimento de software não é constante por parte do cliente, a terceirização passa a ser uma alternativa viável para atender às necessidades. Porém, a questão de terceirizar não é tão simples quanto parece. Controlar qualidade, produtividade e prazo tanto de contratada quanto de contratante sem processos e templates definidos a partir de um modelo de referência, como, por exemplo, MPS.BR ou o CMMI, não é uma tarefa trivial.

A partir da análise do resultado de desempenho do conjunto de fornecedores a Synapsis estará em condições de decidir quais poderão atender cada um dos tipos de projetos conforme características específicas. É essencial a adoção de modelos de referência para orientar a melhoria continua dos processos da organização. O monitoramento de indicadores de desempenho financeiro, qualidade e produtividade, que retratam o desenvolvimento de sistemas de informação, facilita a efetiva gerência dos projetos, produzindo serviços com níveis de qualidade elevado, com o mínimo custo possível - ou seja, alta produtividade - que se constitui em fator crítico de sucesso para o bom desempenho empresarial e aumento das vantagens competitivas das organizações.

\section{Referências}

Chrissis, M.B., Konrad, M., Shrum, S., 2006, CMMI (Second Edition): Guidelines for Process Integration and Product Improvement, Addison Wesley Professional.

Simões, C., Santos, G., Rocha, A.R., 2009, "Implantação do Processo Aquisição na Synapsis Brasil". In: V Workshop Anual do MPS - WAMPS 2009 (WA-MPS.BR), Campinas, SP.

Softex, 2009, "MPS.BR - Guia Geral", SOFTEX, v. MR-MPS: 2009, 10/jan/2010.

Solingen, R., Berghout, E., 1999, The Goal/Question/Metric Method: A Practical Guide for Quality Improvement of Software Development, McGrawHill. 